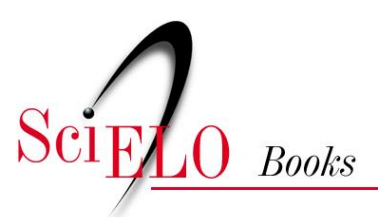

\title{
Régimen multicultural y educación propia en el Ecuador
}

\author{
Sebastián Granda Merchán
}

\section{SciELO Books / SciELO Livros / SciELO Libros}

GRANDA MERCHÁN S. Régimen multicultural y educación propia en el Ecuador. In: GARCÉS, F., and BRAVO, R., eds. Interculturalidad. Problemáticas y perspectivas diversas [online]. Quito: Editorial Abya-Yala, 2019, pp. 185-216. ISBN: 978-9978-10-497-2. https://doi.org/10.7476/9789978104972.0007.

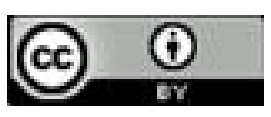

All the contents of this work, except where otherwise noted, is licensed under a Creative Commons Attribution 4.0 International license.

Todo o conteúdo deste trabalho, exceto quando houver ressalva, é publicado sob a licença Creative Commons Atribição 4.0.

Todo el contenido de esta obra, excepto donde se indique lo contrario, está bajo licencia de la licencia $\underline{\text { Creative }}$ Commons Reconocimento 4.0. 


\title{
Régimen multicultural y educación propia en el Ecuador ${ }^{1}$
}

\author{
Sebastián Granda Merchán \\ Universidad Politécnica Salesiana
}

\section{Introducción}

A partir de la década de los 80 del siglo pasado, se instaura en el Ecuador un nuevo modelo de gestión de la diversidad cultural y de procesamiento de las demandas de las organizaciones indígenas, en particular; modelo que se lo ha catalogado de diferentes maneras dependiendo del autor: régimen multicultural (Assies, 1999), multiculturalismo oficial (Wade, 2000), multiculturalismo de Estado (Martínez, 2007), constitucionalismo multicultural (Van Cott, 2000), multiculturalismo neoliberal (Hale, 2007), entre otros.

En términos generales, la implementación de dicho modelo provocó cambios importantes en la relación Estado/pueblos indígenas, así como transformaciones profundas en la forma de organización y vida cotidiana de dichos pueblos. El presente texto tiene como propósito central analizar el impacto que el régimen multicultural tuvo en una

$1 \quad$ El presente texto recupera parte de los resultados de la tesis doctoral La institucionalización de la EIB en el Ecuador y su impacto de las iniciativas de educación propia: reflexionando a partir de la experiencia del Sistema de Escuelas Indígenas de Cotopaxi, desarrollada en el marco del Programa de Doctorado en Estudios Culturales Latinoamericanos de la Universidad Andina Simón Bolívar- Sede Ecuador. El trabajo de campo se realizó en las comunidades indígenas de los alrededores del Quilotoa (Provincia de Cotopaxi), entre el 2013 y el 2014. 
de las dimensiones clave de la vida de las comunidades indígenas de las zonas rurales andinas: la educación.

Para el análisis se ha tomado como referencia la trayectoria y experiencia de una iniciativa de educación indígena emblemática del país: el Sistema de Escuelas Indígenas de Cotopaxi, SEIC; iniciativa impulsada por las comunidades indígenas de las zonas de páramo de la Provincia de Cotopaxi con apoyo del equipo pastoral de Zumbahua, con el objetivo de atender las demandas de formación locales. La pregunta que guía la reflexión es la siguiente: ¿qué nos dice la experiencia en cuestión sobre el modo de operar del multiculturalismo en el ámbito local y sus efectos en las iniciativas de educación propia?

El tema es relevante por cuanto plantea la discusión de la problemática del multiculturalismo y sus efectos desde la perspectiva de experiencias de carácter local, y no a partir del análisis macro de las políticas implementadas por el Estado, que ha sido la tendencia en el país en materia de investigación.

Para el análisis se han considerado los dos momentos del régimen multicultural: aquel de cuño neoliberal y que operó durante las décadas de 1980, 1990 y parte del 2000; y aquel que en la última década se ha convenido en nominarlo como multiculturalismo posneoliberal (Breton \& Martínez, 2015), y que hace alusión al modelo de gestión de la diversidad de los gobiernos de la región que se han identificado como de izquierda.

La hipótesis que se busca argumentar en el texto que sigue es que la implementación del régimen multicultural en el Ecuador, lejos de haber contribuido al desarrollo y fortalecimiento de la educación indígena, terminó propiciando la cooptación y despolitización de las iniciativas de educación propia, proceso que encuentra su explicación de fondo en el modelo de Estado que estuvo en la base de ambos momentos.

Con fines expositivos se ha organizado el texto en cuatro secciones. En la primera se hace una breve referencia a las iniciativas de educación propia en el Ecuador y, de manera particular, a la experiencia del SEIC. En la segunda, se revisa el modo de operar y efectos del régimen multicultural en el Ecuador, considerando sus dos momentos. En la tercera sección se discuten los efectos del régimen multicultural en la forma de organización y dinámica de las iniciativas de educación propia. 
Y, en la última, se reflexiona sobre el modelo de Estado que estuvo en la base de ambos momentos multiculturales.

\section{La educación propia en el Ecuador y el SEIC}

Por educación propia entendemos aquellas iniciativas de educación impulsadas y sostenidas por las mismas comunidades indígenas de las zonas rurales, por cuenta propia o con el apoyo de ciertas alas progresistas de la Iglesia o partidos de izquierda, con el objetivo de atender las demandas de formación locales. En el Ecuador, las iniciativas de educación propia nacen en la década de los 40 del siglo pasado, al calor de la consolidación del proceso organizativo de las comunidades indígenas y la lucha por la tierra.

La primera iniciativa de este tipo fueron las Escuelas Clandestinas de Cayambe impulsadas por Dolores Cacuango y la Federación Ecuatoriana de Indios en 1945, en el marco de la lucha por la tierra, y con el objetivo de alfabetizar a los niños y jóvenes de las comunidades de la zona y dignificar la vida de la población vinculada a las haciendas (González, 2015). Luego está el Proyecto de Educación Bilingüe Autogestionaria de Simiatug liderado por la Fundación Runacunapac Yachana Huasi, que nace a finales de la década de los 60 , con el objetivo de también alfabetizar a los comuneros de la zona de Simiatug y potenciar su proceso organizativo (Rodríguez, 2018).

A estas dos iniciativas se sumaron otras más en diferentes puntos de la Sierra, la Amazonía, e inclusive la Costa ecuatoriana, durante la década de 1970; entre las que destacan el Sistema Educativo Radiofónico Bicultural Shuar y el Sistema de Escuelas Indígenas de Cotopaxi, ambas experiencias apoyadas por la Comunidad Salesiana; el Programa Alternativo de Educación Bilingüe Intercultural de la CONFENIAE, el Programa Educativo Mondayacu Causai Yachana, llevado adelante por una organización de base filial de la Federación de Organizaciones Indígenas del Napo, la Propuesta Educativa Awa Kwaiker, coordinada por la CONAIE, entre otras (Conejo, 2008).

Una característica central de las iniciativas educativas arriba mencionadas es que fueron iniciativas de carácter autogestionario que nacie- 
ron y operaron al margen del Estado, ya sea como una respuesta a la ausencia de escuelas fiscales en las zonas de referencia, o como una alternativa a la educación pública (en aquellas zonas en las que si llegaron las escuelas fiscales) que era vista por la población indígena como una amenaza para su vida y proyecciones como colectividad. Otra característica relevante de aquellas fue su imbricación con los procesos de afirmación cultural y lucha de la población indígena; y, como derivación de lo anterior, su apuesta por formas alternativas de organización del trabajo educativo, tanto a nivel curricular como metodológico y didáctico (Torres, 1992).

Todas estas iniciativas pasaron a formar parte del Estado en 1989, año en el que se institucionaliza la educación indígena y se crea la Dirección Nacional de Educación Intercultural Bilingüe, DINEIB, para que se hicieran cargo de su administración y gestión en el país. Como se verá más adelante, la institucionalización de la educación indígena y creación de la DINEIB forman parte del momento multicultural en el país, y tuvieron repercusiones importantes en la dinámica y forma de organización de dichas iniciativas.

\section{El Sistema de Escuelas Indígenas de Cotopaxi}

Como se adelantó arriba, el SEIC constituye un ejemplo emblemático de educación propia en el país. Constituye un ejemplo emblemático no solo por su amplia cobertura e impacto (llegó a atender las demandas de formación de seis de los siete cantones de la Provincia de Cotopaxi a través de una red de más de 120 instituciones educativas), sino también por su sostenibilidad en el tiempo: operó por cerca de cuatro décadas, con los altibajos del caso.

El SEIC nace en 1975 en los páramos occidentales de la Provincia de Cotopaxi, en lo que actualmente constituyen las Parroquias de Zumbahua, Guangaje y Chugchilán. Fue una iniciativa gestada por los líderes indígenas de la zona, conjuntamente con el apoyo del Equipo Pastoral de Zumbahua; y que buscó atender las demandas de formación de las comunidades.

En la década de los 70, las comunidades en cuestión se encontraban empeñadas en la implementación de un interesante proyecto de desarrollo endógeno, cuyos principales ejes fueron la recuperación de la 
tierra, el control de la comercialización de los productos agropecuarios, y el acceso a espacios de poder local (Guerrero,1992). En el marco de dicho proyecto, el SEIC pasó a cumplir un papel central, pues contribuyó a desarrollar en la población de la zona ciertas habilidades claves, entre las que destacan el aprendizaje de la lectura y la escritura, ${ }^{1}$ el manejo de las operaciones matemáticas básicas y el desarrollo de un pensamiento propio (Herrán, 2008).

En términos del modelo de organización, el SEIC fue una iniciativa de carácter comunitario en todo el sentido de la palabra, pues no solo fue gestada y sostenida por las comunidades, sino - y esto es lo más relevante- fue controlada y direccionada por ellas. Las comunidades eran las que definían el perfil de los educadores y tenían la potestad para seleccionarlos y retirarles del cargo, en caso de que no cumplieran con lo esperado. De igual manera, las comunidades eran las encargadas de definir los objetivos del proceso formativo, así como de hacer seguimiento y validar el trabajo de los educadores en el día a día.

El control que las comunidades tenían sobre el proceso educativo y los educadores, en particular, fue posible gracias al hecho de que fueron las mismas comunidades las que sostuvieron a los educadores, ya sea a través del trabajo de sus tierras o la entrega regular de comida o donaciones en dinero. De esta manera, se aseguró que los educadores respondieran al mandato de las comunidades y cumplieran con lo acordado en las asambleas.

En relación con la propuesta formativa, en estricto sentido, el SEIC se orientó, como se adelantó arriba, a desarrollar las habilidades que el proyecto político del momento demandaba: aprender a leer y escribir, aprender a calcular, y aprender a pensar con cabeza propia. La urgencia de aprender a leer y escribir respondía a la necesidad de los comuneros de no dejarse engañar por parte de los terratenientes y abogados en los procesos de negociación de las tierras. El aprendizaje de las operaciones matemá-

1 El nivel de analfabetismo en la zona en cuestión, en la década de 1970, era alarmante. En 1974, más del 80\% de la población indígena mayor de ocho años era analfabeta, situación que era aún más crítica en el caso de las mujeres (Martínez \& Burbano, 1994). 
ticas, por su parte, se orientaba a evitar los abusos perpetrados por la población mestiza de las zonas aledañas en los procesos de comercialización de los productos agropecuarios (Lazos \& Lenz, s/a).

Por último, el aprender a pensar con cabeza propia ocupaba un lugar central en el proyecto educativo, por cuanto implicaba desarrollar en los comuneros no solo la capacidad para analizar críticamente el contexto local desde parámetros propios, sino también la habilidad para plantear soluciones pertinentes a los problemas que les aquejaba como colectividad.

Para desarrollar las habilidades arriba descritas, la propuesta formativa se estructuró en cinco áreas académicas: Matemática, Ciencias Naturales, Ciencias Sociales, Castellano y Kichwa; cada una de las cuales contaba con sus propios materiales (las cartillas) para el trabajo con los estudiantes (Iñiguez \& Guerrero, 1986). En el día a día, la lengua de enseñanza fue el kichwa y la metodología de trabajo estuvo fuertemente influenciada por los aportes de la Pedagogía de la Liberación de Paulo Freire (Poaquiza, 2013) y los aportes metodológicos de Monseñor Leonidas Proaño.

Desde 1975 hasta 1988, el SEIC operó sin reconocimiento del Estado y, en varias de las comunidades, de manera clandestina. ${ }^{2}$ En 1988, el SEIC fue reconocido por el Estado, y, a partir de 1989, año en el que se oficializó la educación intercultural bilingüe en el Ecuador, el SEIC pasó a formar parte de la jurisdicción de educación intercultural bilingüe, y a responder a las políticas de la DINEIB y su representante en el nivel provincial: la Dirección Provincial de Educación Intercultural Bilingüe de Cotopaxi.

Visto desde la perspectiva de su modelo de gestión y su propuesta formativa, el SEIC constituye una iniciativa que guarda semejanzas con varias experiencias de educación indígena impulsadas en el país y la región. A nivel local, el SEIC mantiene varios elementos en común con

2 Operaron de manera clandestina por la experiencia negativa que tuvieron con los hacendados de la zona, que no vieron con buenos ojos el nacimiento de las escuelas ni que los indígenas se educaran. 
las Escuelas Clandestinas de Cayambe o con las Escuelas Autónomas de Simiatug, por ejemplo. En términos regionales, la iniciativa comparte varias similitudes con las escuelas Zapatistas (Baronet, 2012) y el proyecto educativo del Consejo Regional Indígena del Valle del Cauca, en Colombia (CRIC, 2004).

\section{El régimen multicultural en el Ecuador}

El multiculturalismo constituye un modelo particular de conceptualizar el tema de la diversidad cultural pero también de intervenir en ella y gestionarla. En tanto que modelo conceptual, el multiculturalismo es un enfoque de carácter descriptivo que reconoce a los diferentes grupos culturales que coexisten en un territorio determinado (ya sea a nivel local, nacional o internacional), pero haciendo caso omiso de las jerarquías y relaciones de poder que se establecen entre ellos. En tanto que modelo de gestión, es un modelo que apela a la tolerancia de las diferencias y a su inclusión en la sociedad mayor, pero sin poner en cuestión, y menos aún en riesgo, la estructura de aquella y sus parámetros de funcionamiento (Walsh, 2009).

El tema de la inclusión es central para este modelo y constituye uno de los elementos que lo diferencian de otros modelos de gestión de la diversidad (Dussel, 2007). Ciertamente, a diferencia de la interculturalidad, por ejemplo, que apuesta por la re-estructuración de la sociedad y sus instituciones con el objetivo de propiciar la igualdad entre los grupos culturales, su encuentro e intercambio de experiencias y saberes; el multiculturalismo aboga por la incorporación de lo diferente en la sociedad e instituciones establecidas, proceso en el que los grupos excluidos terminan, por lo general, ocupando lugares periféricos y devaluados.

Al referirse a la política de la liberación, Dussel utiliza una metáfora interesante que da luces sobre el modo de operar del multiculturalismo y sus diferencias con proyectos orientados a transformar la sociedad:

No se trata de un proceso de inclusión, sino de creación novedosa, analógica, transformadora. No es cuestión de hacer simplemente una nueva habitación para los excluidos en la antigua casa. Es necesario hacer una nueva casa, con nueva distribución, de lo contrario los indígenas, 
las mujeres y los afroamericanos irán a las habitaciones "de servicio" ... como antes, como siempre. (Dussel, 2007, p. 137)

Lo anterior explica por qué para varios autores, ${ }^{3}$ el multiculturalismo constituye un modelo conceptual y de gestión de la diversidad cultural que todavía mantiene una perspectiva colonial; un modelo que lejos de propiciar la transformación de la sociedad, en aras de una mayor igualdad y equidad entre los grupos culturales que cohabitan en un espacio determinado, termina reproduciendo las jerarquías y relaciones de dominación que se han desarrollado entre ellos.

El multiculturalismo es un modelo que tiene sus raíces en la experiencia de los países del norte por incluir a los grupos minoritarios y migrantes, y tiene una larga historia de desarrollo teórico por parte de la academia de dichos países. Al momento, cuenta con varios enfoques teóricos, siendo el enfoque liberal — con el filósofo canadiense Will Kimlicka a la cabeza- el que más impacto ha tenido en la definición de políticas para los pueblos indígenas y afrodescendientes en América Latina.

En el Ecuador, la implementación de dicho modelo tuvo lugar a inicios de la década de los 80 del siglo pasado, con el retorno a la democracia. Ya en 1979, el primer presidente del nuevo ciclo democrático, Jaime Roldós Aguilera, incluyó en su discurso de posesión unas palabras en kichwa y reconoció el carácter pluriétnico y pluricultural del país. Tiempo después, se creó la Secretaría de Desarrollo Rural Integral, con la finalidad de atender las demandas de la población rural, e impulsó un ambicioso programa de escolarización y alfabetización para la población rural e indígena (Ortiz, 2016).

Sin embargo, fue en el gobierno de Rodrigo Borja que el régimen multicultural se expresó en toda su esencia, con la creación de la Dirección Nacional de Educación Intercultural Bilingüe como instancia encargada de la administración de la educación indígena en el país, y el reconocimiento y titulación parcial de territorios en la Amazonía

3 En esta línea de reflexión, son importantes los aportes de Walsh (2009), López (2019) y del mismo Dussel (2007). 
ecuatoriana, en respuesta a la demanda de la Organización de Pueblos Indígenas de Pastaza (Pallares, 2007).

$\mathrm{Al}$ igual que en otros países de la región, la apertura hacia la diversidad cultural en el Ecuador fue el resultado de la imbricación de varios procesos que se dieron de manera simultánea y, muchas de las veces, en tensión: la implementación del modelo neoliberal y sus respectivas políticas de ajuste, el retorno al régimen democrático luego de varios períodos de gobiernos militares, la crisis fiscal y de legitimidad del Estado, y la emergencia de un movimiento indígena fuerte, que desde mediados de la década de los 80 puso en cuestión la matriz de organización del Estado y la sociedad (Assies, 1999).

Este aspecto es fundamental considerar por cuanto contribuye a comprender el alcance reducido, en materia redistributiva, del régimen multicultural neoliberal, en general, y de sus políticas en los diferentes ámbitos del devenir social, en particular. Y es que fueron políticas que se implementaron en el contexto del repliegue del Estado en áreas claves del desarrollo económico y social, y de un giro importante en sus prioridades de intervención y forma de operar.

Si se quisiera hacer una síntesis de su forma de actuar en el nivel macro, se podría argumentar que el multiculturalismo, en el contexto neoliberal, operó a través de tres estrategias centrales: el reconocimiento jurídico de la diversidad cultural, la creación de instancias de gobierno especiales para la gestión de las problemáticas indígenas, y la inclusión de representantes indígenas en el Estado y sus diferentes instituciones (Larrea, 2010; Walsh, 2009); todas ellas implementadas, como se señaló arriba, en el marco del paradigma de la inclusión.

¿Cuál es el balance que se puede hacer del impacto del modelo multicultural de cuño neoliberal luego de casi dos décadas y media de operación: 1980-2006? Con Larrea (2010) podemos sostener que su impacto en el país no fue para nada positivo, pues su implementación garantizó no solo la aplicación de políticas neoliberales duras en el agro y en las zonas rurales, con todo lo que aquello significó en términos de desarticulación de las comunidades y deterioro de sus condiciones de vida, sino que contribuyó a desmontar las demandas más radicales de 
las organizaciones indígenas (aquellas orientadas a la transformación de la estructura económica) y a debilitar su capacidad de movilización.

Hasta aquí en lo que tiene que ver con el multiculturalismo de cuño neoliberal. El multiculturalismo posneoliberal, por su parte, es un régimen que se ha ido consolidando en la región durante la última década y media, a partir de la entrada en el escenario político de gobiernos que se han catalogado a sí mismos como de izquierda.

En el caso del Ecuador, en particular, el multiculturalismo posneoliberal fue el resultado de varios cambios importantes, entre los que destacan los siguientes: la apuesta por un nuevo modelo de desarrollo que buscó dejar atrás los elementos centrales del neoliberalismo, el retorno del Estado como instancia rectora del desarrollo, regulación de la economía y redistribución de los ingresos; y el debilitamiento del movimiento indígena y pérdida de protagonismo en el escenario político.

Si se compara el régimen multicultural posneoliberal con el neoliberal se pueden constatar algunas diferencias importantes en cuanto a su forma de operar. La primera diferencia tiene que ver con la manera de procesar el tema de las diferencias en el nivel discursivo. El multiculturalismo posneoliberal es un modelo que ha reconocido no solo la diversidad cultural existente en el territorio nacional, sino también las jerarquías y asimetrías que le son consustanciales. Consecuente con lo anterior, es un régimen que ha proyectado para el mediano y largo plazo nuevos formatos de organización del Estado y la sociedad que propician la igualdad. La alusión reiterada al Estado plurinacional y al Sumak Kawsay en los discursos y planes de desarrollo de la última década, da cuenta de lo anterior.

Conectado con el tema anterior, se encuentra el aspecto normativo. El régimen posneoliberal ha promovido avances importantes en materia jurídica, de manera especial a nivel de los contenidos de la normativa mayor, como es el caso de la Constitución Política, así como de ciertas leyes secundarias. En esta línea, es destacable, por ejemplo, el reconocimiento del Ecuador como un Estado plurinacional e intercultural, además de democrático, soberano, independiente, etc.; así como la ampliación y fortalecimiento de los derechos colectivos de las nacionalidades y pueblos indígenas (Grijalva, 2008). 
Una tercera diferencia hace referencia al ámbito de la autonomía y participación. A diferencia del multiculturalismo de cuño neoliberal, el multiculturalismo posneoliberal ha tendido a excluir a los pueblos indígenas de la definición y gestión de aquellas políticas que inciden de manera directa en su vida. En esta línea, coincidimos con Breton y Martínez (2015) en que dicho modelo ha significado un retroceso a nivel de la relación Estado/pueblos indígenas, y que se ha retornado al momento anterior al neoliberalismo, en el que el Estado actuaba en nombre de los pueblos indígenas y en el que estos últimos constituían simples receptores pasivos de las políticas.

La última diferencia hace alusión a la forma de interpelación y naturaleza de las políticas públicas: en el contexto del multiculturalismo posneoliberal el Estado ha interpelado a la población indígena como ciudadanos pobres (con todo lo que ello implica) y no como nacionalidades y pueblos, y ha optado — como derivación de lo anterior - por la implementación de políticas públicas de carácter universal para atender sus demandas.

El balance del impacto del régimen posneoliberal en materia de mejoramiento de las condiciones de vida y empoderamiento de los pueblos y nacionalidades indígenas tampoco es halagador y tiene tintes contradictorios, pues a la par de que se ha incrementado la asignación de recursos públicos para dichos sectores, lo cual ha propiciado el mejoramiento de varios indicadores relacionados con sus condiciones de vida, se han desmantelado los espacios de autonomía alcanzados en las décadas anteriores y debilitado el control que tenían sobre ciertos recursos claves para su sobrevivencia; a lo que se suma la desarticulación de sus organizaciones y capacidad de movilización por efecto de la cooptación y criminalización de varios de sus líderes (León, 2010; Martínez, 2010).

\section{El multiculturalismo y las iniciativas de educación propia}

Hasta aquí se ha dado una visión general sobre el modo de operar del régimen multicultural en el Ecuador y su impacto en la población indígena. Ahora bien, ¿qué significó el régimen multicultural para las experiencias de educación indígena de carácter local, y, de manera par- 
ticular, para aquellas iniciativas que fueron generadas y sostenidas por las mismas organizaciones indígenas? ¿Qué cambios trajo la implementación del régimen multicultural en la estructura y forma de funcionamiento de dichas iniciativas?

En base a la experiencia del SEIC, que comparte varios elementos en común con otras experiencias educativas que tuvieron lugar en diferentes puntos del país, podemos decir que el multiculturalismo, lejos de haber propiciado el fortalecimiento de las iniciativas de educación indígena (en los términos manejados por las mismas organizaciones indígenas), significó un proceso de cooptación de aquellas por parte del Estado y su despolitización. Pasemos a revisar con detenimiento el tema, manteniendo la diferenciación de los dos momentos a los que se hizo alusión anteriormente.

Como se adelantó arriba, el multiculturalismo neoliberal significó, en materia educativa, el reconocimiento de la educación indígena como una educación diferenciada, y, sobre la base de lo anterior, la creación de una instancia especializada dentro del Estado para su gestión y administración: la DINEIB, instancia controlada por representantes de las mismas organizaciones indígenas.

En el nivel provincial, el nuevo formato institucional supuso la creación de instancias especializadas de gestión de la educación indígena: las direcciones provinciales de educación intercultural bilingüe, también controladas por las organizaciones indígenas y con potestad para definir y regular aspectos centrales del día a día de la educación en las comunidades indígenas: los docentes que ingresaban a las escuelas y salían, el proceso de formación de los docentes, el currículo, los materiales y recursos, entre otros.

Desde una perspectiva panorámica, se puede sostener que el nuevo modelo de organización de la educación indígena generado en el contexto neoliberal trajo cuatro cambios importantes en el ámbito local y en la dinámica de las iniciativas de educación propia: la estatización de dichas iniciativas, la enajenación del control de las comunidades, la despolitización de los proyectos educativos, y su capitalización simbólica. En lo que sigue se hará referencia a cada uno de estos cambios. 


\section{Estatización de las iniciativas}

La implementación del régimen multicultural en el Ecuador implicó un lento pero irreversible proceso de estatización de las iniciativas de educación propia. Como se señaló arriba, las experiencias de educación propia nacieron al margen del Estado y por iniciativa de las mismas comunidades indígenas, en un contexto de serios problemas económicos y sociales, por un lado, y de activación política de aquellas, por otro. Debido a lo anterior, fueron experiencias que contaron con un amplio margen de autonomía y movilidad para adecuarse a las demandas de formación de las comunidades.

La implementación del régimen multicultural en el país significó la ampliación del radio de acción del Estado en el contexto rural y la apropiación de las iniciativas de educación indígena. Decimos "ampliación del radio de acción" y no "intervención” por cuanto el Estado ha estado presente en la vida cotidiana de las comunidades indígenas desde mucho tiempo atrás, a través de diferentes instituciones y canales. Con la creación de la DINEIB y las direcciones provinciales, todas las iniciativas de educación propia pasaron a formar parte del Estado y a sujetarse a sus directrices, dinámicas y tiempos.

Lo interesante de la experiencia ecuatoriana es que dicho proceso de sujeción tuvo lugar en un contexto en el que la institucionalidad estatal encargada de la educación indígena, la DINEIB, estuvo en manos de las mismas organizaciones indígenas, una de las características centrales del régimen multicultural neoliberal, como se pudo apreciar a arriba.

Lo anterior explica por qué el proceso de estatización fue asumido y experimentado como un proceso positivo por parte de las comunidades indígenas y sus respectivas organizaciones y, de la mano con esto, por qué dicho proceso se dio de una manera fluida y sin tensiones mayores en los primeros momentos. Fue en el momento en que las políticas de la DINEIB y las direcciones provinciales comenzaron a alterar la dinámica cotidiana de las escuelas y a desdibujar su forma de organización y trabajo, que iniciaron las tensiones entre las comunidades y el Estado. 


\section{Enajenación del control de las iniciativas educativas}

Conectado con el tema anterior se encuentra el tema del control. El nuevo formato institucional de la educación indígena en el Ecuador significó no solo la sujeción de las iniciativas educativas al Estado, sino también la enajenación del control de aquellas.

Las iniciativas de educación propia, por el hecho de haber sido impulsadas por las mismas comunidades de las zonas rurales, fueron iniciativas cuyo control reposó en aquellas. Eran las mismas comunidades indígenas las que tenían la potestad para seleccionar a los educadores, para pedirles cuentas de manera regular, así como para prescindir de sus servicios si no cumplían con lo estipulado. De igual manera, eran las comunidades las que tenían la potestad para definir lo que se debía trabajar en el aula y cómo hacerlo.

La implementación del modelo multicultural en el país significó la pérdida del control de las comunidades sobre la educación y su traspaso al Estado. Ahora bien, el proceso de enajenación del control de la educación no se dio por la fuerza sino como resultado de un acontecimiento puntual: el ingreso de los educadores comunitarios al magisterio y su articulación al sistema de educación nacional. El momento en que pasaron a formar parte del magisterio y a ser pagados por el Estado, los educadores comunitarios pasaron a seguir sus directrices y a rendir cuentas a aquel, y ya no a las comunidades.

Es importante destacar, sin embargo, que en el marco de la nueva institucionalidad y forma de organización de la educación indígena, no todos los ámbitos de decisión sobre la educación pasaron a manos del Estado, pues algunos de ellos quedaron en manos de las comunidades, aspecto que contribuye a comprender por qué ciertos aspectos centrales de los proyectos de educación propia se mantuvieron durante varios años a pesar de su articulación con el Estado: nos referimos, por ejemplo, a la definición de la propuesta curricular, por ejemplo, pero también a procesos centrales como la capacitación de los educadores.

La situación anterior se explica no tanto por una supuesta naturaleza condescendiente o benévola del Estado, sino por la presencia, en un primer momento, de representantes de las organizaciones indíge- 
nas en la institucionalidad estatal que optaron, a ratos por convicción y a ratos por falta de fuerza para intervenir, por respetar cierto nivel de autonomía de las comunidades para la toma de algunas decisiones en materia educativa; $y$, en un segundo momento, por efecto de los proyectos de descentralización educativa que se implementaron en las zonas rurales como parte de la agenda neoliberal (Ponce, 2010).

\section{Despolitización del proyecto educativo}

Los cambios en materia institucional generados en el contexto neoliberal significaron no solo la estatización y la enajenación de control de las iniciativas educativas comunitarias, sino también, y como consecuencia de estos últimos, un proceso de despolitización de los proyectos educativos comunitarios. El hecho de pasar a formar parte del Estado y de pasar a estar controlados por aquel, provocó un proceso de simplificación de los proyectos educativos comunitarios, por efecto del cercenamiento de su arista más política. Expliquemos la problemática.

En el período anterior a la incorporación de las iniciativas educativas al Estado, los educadores comunitarios terminaron convirtiéndose en los verdaderos líderes de las comunidades y las escuelas comunitarias en las instancias encargadas de aglutinar y organizar a las comunidades, e impulsar sus proyectos. Fue desde las escuelas, y bajo el liderazgo de los educadores comunitarios, desde donde se organizó la toma y recuperación de las tierras, por ejemplo, pero también desde donde se gestionaron proyectos de diferente índole que se requerían en las comunidades: agua, criaderos, tiendas comunales, proyectos productivos, etc.

El momento en que los educadores comunitarios pasaron a formar parte del magisterio y del sistema educativo nacional, aquellos fueron perdiendo paulatinamente el liderazgo en sus respectivas comunidades y las escuelas la capacidad para organizar a las comunidades y llevar adelante sus proyectos. Con el paso de los años, las escuelas comunitarias se redujeron a instancias estrictamente de formación de las nuevas generaciones, en el sentido clásico de la palabra.

Es importante destacar, sin embargo, que el proceso de despolitización de las iniciativas educativas no fue un proceso total sino parcial, 
pues a pesar de que los proyectos educativos originales fueron reducidos a su componente de educación formal, aquellos buscaron responder a las demandas y aspiraciones de la población de la zona que, para el momento, consistía en desarrollar los conocimientos y habilidades necesarios para que las nuevas generaciones pudieran emprender en diferentes áreas productivas, evitando la migración a las ciudades y su inserción en el mercado laboral en condiciones desfavorables.

\section{Capitalización simbólica de las iniciativas}

Para cerrar este apartado, es necesario destacar un cuarto elemento de impacto del régimen multicultural y que tiene que ver con el tema del reconocimiento de las iniciativas. En efecto, fue en el marco del régimen multicultural de cuño neoliberal que las iniciativas educativas comunitarias fueron reconocidas por el Estado como propuestas válidas y legítimas, aspecto que, sin lugar a dudas, contribuyó a su sostenibilidad en el tiempo y a dotarlas de capital simbólico.

Con el paso de los años, las iniciativas educativas comunitarias ganaron terreno en el imaginario de la población de las zonas rurales, y terminaron convirtiéndose en la mejor opción educativa de las comunidades indígenas, inclusive en aquellas zonas en las que la escuela indígena competía con la escuela hispana y en las que se impulsó una fuerte campaña de desprestigio de las escuelas indígenas. El problema es que, como se discutió arriba, el reconocimiento y el proceso de capitalización simbólica de las iniciativas de educación indígena se dio a expensas de su metamorfosis y despolitización.

Apelando a la metáfora de Hale (2007), se puede afirmar que el principal efecto del multiculturalismo neoliberal en el ámbito educativo local, fue la construcción de un modelo de educación indígena permitido, como resultado de dos procesos que se dieron de manera simultánea: el reconocimiento y legalización de las iniciativas de educación indígena comunitaria, por un lado, y el cercenamiento de su arista más política, por el otro; un modelo que si bien mantuvo algunos de los elementos originales, dejó — con el paso de los años— de ser una amenaza para el orden local estatuido. 
Hasta aquí lo que tiene que ver con el multiculturalismo neoliberal, pero ¿qué ocurrió con las iniciativas educativas en el contexto del multiculturalismo posneoliberal? El multiculturalismo posneoliberal fue un régimen de gestión de la diversidad que, a contracorriente con su discurso totalmente a favor de la diversidad y la interculturalidad, terminó desmantelando el sistema de educación intercultural bilingüe construido en el momento neoliberal, y, como consecuencia de lo anterior, cercenando el control que las organizaciones indígenas tenían sobre la educación.

El desmantelamiento del sistema de educación intercultural bilingüe supuso no solo la erradicación de la DINEIB (en tanto que instancia con capacidad de decisión y gestión de la educación indígena), sino también el cierre de sus brazos operativos en el nivel local: las direcciones provinciales. Producto de estos cambios, la educación indígena pasó a manos del ministro de educación y su equipo de trabajo, y a responder a las políticas y directrices estándar del momento.

En términos generales, se puede sostener que los cambios producidos en materia institucional y de lineamientos en materia educativa en el contexto posneoliberal provocaron cuatro giros fundamentales en las iniciativas de educación indígena locales: la enajenación total de su control, el reforzamiento del control y vigilancia externos, la estandarización del modelo educativo, y su descapitalización simbólica. Revisemos rápidamente cada una de ellas.

\section{Enajenación total del control de las iniciativas educativas}

Como se discutió en el apartado anterior, en el contexto del multiculturalismo de cuño neoliberal algunos ámbitos de decisión educativa quedaron en manos de las comunidades indígenas, aspecto que contribuyó a mantener ciertos aspectos centrales de los proyectos, como fue el proceso de definición de la propuesta formativa, y, en un segundo momento, el proceso de selección de los docentes y su capacitación. En el marco del régimen multicultural posneoliberal aquello concluyó de una manera radical, pues todos los ámbitos de decisión pasaron a manos del ministro de educación, y a implementarse desde las instancias de gobierno descentralizadas: las direcciones zonales y distritales. 
Dicho proceso tuvo lugar en el marco de la apuesta del Gobierno del momento por recuperar la rectoría del Estado en materia educativa, pero también en el marco de una fuerte crisis y debilitamiento del movimiento indígena, conjugado con una fuerte campaña de desacreditación de la educación intercultural bilingüe. En el nuevo contexto, los educadores comunitarios y las familias fueron llamados a allanarse a las disposiciones emitidas desde el ministerio de educación y a encauzar su participación en materia educativa a través de los canales diseñados para el efecto.

A diferencia del proceso de enajenación que se vivió en el período anterior, este proceso de enajenación fue vivido con mucho malestar por los líderes y educadores comunitarios, pues fue asumido como una usurpación de la posibilidad de impulsar proyectos educativos que consideraran no solo las características de los niños y jóvenes indígenas, sino también la realidad y proyectos de las comunidades.

En el nuevo contexto, el margen de movilidad de los directivos y docentes se redujo radicalmente y la posibilidad de impulsar prácticas alternativas que respondieran a la realidad de los estudiantes y la zona pasó a depender de la buena voluntad de los docentes dentro de sus horas de clase. Esto fue lo que sucedió, por ejemplo, con el uso de lengua materna en el proceso de enseñanza-aprendizaje, pero también con la enseñanza de aspectos clave de la vida comunitaria: su historia y forma de organización, las prácticas agropecuarias, conocimientos locales, entre otros.

\section{Reforzamiento de la vigilancia y control externos}

Conectado con el punto anterior, está el tema del control y la vigilancia. La apuesta del gobierno por recuperar la rectoría del Estado, más los cambios que se dieron en materia institucional y organización de la educación, tuvo como resultado un reforzamiento de la vigilancia y control de las escuelas indígenas por parte del Estado, aspecto que se convirtió en una condición central para la implementación de los cambios e innovaciones ofrecidos.

El reforzamiento de la vigilancia y control se dio a través de diferentes vías, siendo las dos más utilizadas las visitas periódicas a las escuelas por parte de los funcionarios de las direcciones distritales y el 
pedido continuo y recurrente de información. Esta última estrategia fue relevante por cuanto a través de ella se aseguró que los directivos y educadores estuvieran al día con las nuevas disposiciones y se acoplaran a las nuevas regulaciones del trabajo escolar.

Demás está decir que el reforzamiento de la vigilancia y control contribuyó a disciplinar a los directivos y docentes de las escuelas indígenas, y a alinearles con el nuevo proyecto educativo del Estado. Esto, junto con la presión provocada por la evaluación del desempeño docente (con instrumentos diseñados para la educación hispana), terminó quebrando la rutina con la que los directivos y los educadores venían operando por años, y provocando malestar en ambos actores.

\section{Estandarización del modelo educativo}

A diferencia del momento neoliberal en el que las iniciativas de educación indígena locales lograron mantener ciertos elementos de los proyectos originales, y en el que se pudo atender algunas de las demandas de las comunidades en materia de formación; en el marco del multiculturalismo posneoliberal se vivió un proceso de estandarización de dichas iniciativas, con miras a alinearlas con el proyecto del Estado.

Dicho proceso fue el resultado de dos cambios que se dieron de manera simultánea: el desmantelamiento de los proyectos educativos originales, por un lado, y la imposición en las zonas rurales del proyecto educativo de la educación hispana, por otro. Cuando hacemos referencia al proyecto educativo de la educación hispana, nos referimos al proyecto de Actualización y fortalecimiento curricular de la educación básica, que tenía como objetivo central elevar la calidad de la educación básica y alinearla con los objetivos del plan de desarrollo del momento.

En la práctica, el proceso de estandarización de las iniciativas educativas y su alineación con el proyecto del Estado, significó cambios importantes en el día a día del trabajo en el aula, entre los que destacan los siguientes: el pasar a trabajar los contenidos de la educación hispana con sus respectivos textos escolares, el pasar a trabajar en castellano, así como la llegada a las zonas rurales de docentes externos a las comunidades que no manejaban la lengua de la zona y que poco o nada conocían de la realidad de aquellas. 
En el marco de este proceso, fue relevante el tema del cierre de las escuelas comunitarias, pues más allá de las repercusiones que aquello tuvo en la vida cotidiana de las comunidades, fue una política que contribuyó a imponer el proyecto educativo de la educación hispana: con el cierre de las escuelas, muchos de los estudiantes pasaron a las Unidades Educativas del Milenio, unidades a través de las cuales el gobierno de turno apostó implementar todos los cambios e innovaciones ofrecidos.

Apelando a la terminología foucaultiana, y mirando los cambios desde una perspectiva panorámica, se podría argumentar que el régimen multicultural de impronta posneoliberal terminó, vía el desmontaje de lo que quedaba de los proyectos educativos originales, normalizando de manera radical las iniciativas de educación indígena de las zonas rurales, con miras a funcionalizarlas a los requerimientos del Estado.

El problema está en que dicho proceso de normalización nunca provocó la tan ansiada funcionalización de las iniciativas de educación indígena locales, pues por efecto de varios factores (dificultad para procesar los contenidos del currículo de la educación hispana, dificultad para enseñar y aprender en castellano, desconocimiento de los nuevos docentes del contexto y la realidad de los educandos, etc.), el nuevo proyecto educativo no logró calar en el día a día de las escuelas, ni potenciar el trabajo cotidiano en el aula.

\section{Descapitalización simbólica}

Para cerrar este apartado es necesario hacer alusión al tema de la descapitalización simbólica de la educación indígena. El gobierno de turno, en su afán por tomar el control de la dinámica educativa en las zonas rurales, llevó adelante una fuerte campaña de desacreditación de la educación indígena a través de diferentes canales. Los argumentos centrales para la desacreditación tenían que ver, fundamentalmente, con la baja calidad de la educación indígena y su sobre-politización, problemática esta última que se manifestaba, según el mismo gobierno, en los contenidos plasmados en los textos escolares producidos por la DINEIB.

Fruto de esta intensa desacreditación, la educación indígena sufrió una pérdida de prestigio importante tanto a nivel nacional como 
en el ámbito local. La pérdida de prestigio de la educación indígena, sumada a las ofertas que el gobierno hizo en materia de educación para la zonas rurales usando a las Unidades Educativas del Milenio como referente central, terminó cambiando la perspectiva de una buena parte de las familias indígenas: durante los años en los que se produjo el desmantelamiento del sistema de educación intercultural bilingüe, era común escuchar en las comunidades indígenas voces en contra de la educación indígena implementada desde tiempo atrás, apelando fundamentalmente a su baja calidad.

Con el paso de los años, muchas de las familias indígenas comenzaron a dudar no solo de la posibilidad de que el Estado llegara a cumplir con lo ofrecido, sino también de que los ofrecimientos iban a mejorar la educación en la zona. Recordemos que, para fines del 2014, un número importante de escuelas comunitarias fueron cerradas y muchos de los niños de aquellas se vieron obligados a pasar a las Unidades Educativas del Milenio, mientras que otros tuvieron que migrar con sus padres para estudiar en las ciudades. Recordemos, también, que para esa época se comenzó a trabajar con el nuevo currículo, el de la educación hispana, y que el mandato era que se trabajara en castellano.

Más allá de los cambios producidos a nivel del imaginario de la población ecuatoriana, en general, y de la población indígena, en particular, es importante destacar que en el contexto del multiculturalismo posneoliberal se dio una vuelta más de tuerca al proceso de descapitalización simbólica de las iniciativas de educación indígena realmente existentes, proceso que tuvo como contraparte la idealización de un nuevo modelo de educación indígena, lleno de todas las virtudes y bondades, pero que hasta fines del 2014 no se había concretado en ninguna provincia del país.

Si se realiza una valoración general del impacto que el multiculturalismo posneoliberal ha tenido en las iniciativas de educación indígena locales, se puede sostener que, a diferencia del multiculturalismo neoliberal que terminó construyendo en las zonas rurales un modelo de educación indígena permitido, el régimen multicultural de impronta posneoliberal terminó imponiendo en dichas zonas el proyecto educativo de la población blanco-mestiza de las zonas urbanas; situación alarmante si se con- 
sidera que fue en el contexto posneoliberal en el que más se avanzó en materia de reconocimiento de los derechos de los pueblos indígenas y en la conceptualización de un nuevo modelo de sociedad y Estado.

Se podría pensar que el proceso de estandarización educativa arriba aludido tuvo como contraparte un re-direccionamiento importante de recursos públicos hacia las escuelas comunitarias, por efecto de las políticas de carácter redistributivo; pero la realidad no fue así. En el 2014, la situación de las escuelas comunitarias en materia de infraestructura y equipamiento era la misma, cuando no peor, que, en el momento neoliberal, con un elemento añadido: que ahora las escuelas estaban prohibidas de buscar, por su cuenta, recursos financieros de la cooperación internacional.

\section{Modelo de Estado y educación indígena}

En las páginas anteriores se ha analizado los cambios que las iniciativas de educación propia vivieron en los dos momentos multiculturales. Ahora bien, hay un elemento común en ambos momentos y que explica buena parte de lo que terminó ocurriendo con dichas iniciativas, a saber: la poca o nula capacidad (dependiendo del momento) de las comunidades indígenas para controlar y dirigir sus iniciativas educativas; aspecto que nos remite a la problemática del modelo del Estado que estuvo en la base de ambos momentos.

En términos generales, se puede sostener que el modelo de Estado que estuvo en la base de los dos momentos multiculturales es el mismo modelo que ha operado, con diferentes matices, a lo largo de toda la época republicana del país: el Estado de impronta mono-cultural y colonial. Expliquemos el tema.

En el Ecuador, al igual que en otros países de la región, se construyó un Estado de carácter mono-cultural y colonial que se fundamentó en los principios de la filosofía liberal y que se sustentó, consecuente con lo anterior, en la figura de la ciudadanía individual (Guerrero, 1994). Dicho modelo de Estado no solo contribuyó a viabilizar y legitimar los procesos de dominación y explotación de la población indígena, sino también a segregarla y excluirla en términos políticos. 
En relación al último punto es fundamental señalar, con Tapia (2007) y Díaz-Polanco (1998), que el formato liberal de Estado que se impuso en el Ecuador contribuyó no solo a coartar la participación de la población indígena en las decisiones de carácter nacional, sino también, y esto es lo más relevante, a anular de raíz toda posibilidad de autogobierno y desarrollo autónomo por parte de dicha población. ${ }^{4}$

Lo anterior explica por qué una de las demandas centrales del movimiento indígena ecuatoriano, desde su emergencia en 1990, haya sido el tema del autogobierno, y, como condición de aquello, la re-estructuración del Estado. Para el movimiento indígena, el autogobierno constituye no solo un derecho que le asiste a la población indígena por su condición de pueblos con una cultura propia y pre-existentes al Estado ecuatoriano, sino también una condición básica, junto con la tierra y el territorio, para su reproducción como colectividades (CONAIE, 2007).

Es este modelo de Estado el que ha estado en la base del régimen multicultural tanto de cuño neoliberal como posneoliberal, un formato en el que no hay lugar para que la población indígena se haga cargo de aquellas dimensiones centrales de su reproducción como pueblos, ni tampoco posibilidad alguna para que cuando una de dichas dimensiones pase a formar parte del Estado (como fue el caso de educación), ésta continúe bajo el control de aquella.

Es en este sentido que podríamos hablar, recuperando uno de los conceptos centrales de Díaz-Polanco (2006), del Estado ecuatoriano como un Estado etnófago, esto es, un Estado que ha operado en base a la cooptación y re-funcionalización de todas aquellas instituciones, procesos y dinámicas de la sociedad que han ido cayendo dentro de su radio

4 Para el liberalismo de viejo cuño, la libertad y la autonomía constituyen potestad únicamente de los individuos y no de los grupos, aspecto que explica su aversión a cualquier demanda enarbolada en nombre del grupo o la cultura del grupo. Fue recién en el transcurso del siglo $\mathrm{XX}$, que el liberalismo reconoció el derecho de los pueblos a la libre determinación, pero en el marco de la construcción de los Estado-naciones independientes; derecho que alimentó y estuvo en la base de los procesos independentistas de los países colonizados de África, Asia y América Latina, posteriores a la Segunda Guerra Mundial (Díaz-Polanco, 1998). 
de acción. Una lógica de operación que, si bien ha impactado en todos los grupos de la sociedad, ha tenido efectos devastadores en los pueblos indígenas por cuanto su vida cotidiana se ha estructurado en base a parámetros radicalmente distintos a los del Estado liberal.

La discusión anterior plantea la problemática de las condiciones de posibilidad para llevar adelante iniciativas de educación alineadas con los intereses y proyectos de las comunidades indígenas. La pregunta que surge, entonces, es la siguiente: ¿es posible pensar en procesos de educación indígena que formen parte del Estado y que, a la vez, respondan a las demandas y proyectos políticos de los contextos locales, o, el hecho de pasar a formar parte del Estado supone, necesariamente, la cooptación y desmantelamiento de dichas iniciativas? En caso de que la primera opción fuera la más factible ¿cuáles serían las condiciones básicas para que aquello se concrete? En caso de que la segunda fuera la opción más realista ¿se debería pensar en propuestas de educación de carácter autónomo, al estilo de la iniciativa de los Zapatistas en México?

De entrada, podemos decir que no vemos factible que en el Ecuador actual se desarrollen propuestas de educación indígena de carácter autónomo, al estilo de los Zapatistas, no solo porque la historia de la pueblos indígenas en el Ecuador y su relación con el Estado ha sido diferente a la situación mexicana, sino también porque una de las demandas del movimiento indígena, desde su emergencia en la década de los 90, ha sido el participar en el Estado y, cuando ha sido posible, el pasar a formar parte de su estructura (Martínez, 2004).

Sostenemos lo anterior a sabiendas de que en los últimos años se han levantado planteamientos desde varias organizaciones indígenas y educadores de escuelas indígenas comunitarias que abogan por volver a construir propuestas de educación al margen del Estado, al estilo de los primeros momentos de la educación indígena en el país. ${ }^{5} \mathrm{Si}$ bien constituye un planteamiento legítimo, consideramos que es una propuesta de

5 En esta línea se encontraban, por ejemplo, el presidente del Movimiento Indígena y Campesino de Cotopaxi y también los directivos y educadores de las escuelas que formaban parte del SEIC. Todos fueron muy enfáticos en este planteamiento, pues en el contexto de las políticas del Gobierno de la Revolución Ciudadana la veían 
difícil concreción si se considera, entre otros factores, la debilidad de las organizaciones indígenas en el momento actual.

A partir de la experiencia analizada y de otras que ha tenido lugar en la región y que mantienen vigencia hasta el día de hoy, consideramos que si es posible pensar en iniciativas de educación que formen parte del Estado y que, a la vez, sean sensibles con las características de la población y respondan a sus necesidades y proyectos colectivos. La condición básica de aquello, sin embargo, es que se asegure el control de la educación por parte de las comunidades indígenas, pues no puede haber proyectos educativos pertinentes sin la participación de la población indígena beneficiaria de aquellos y sin capacidad para definir elementos centrales de la propuesta educativa como, por ejemplo, el currículo, el perfil de los docentes, entre otros.

Con el planteamiento anterior nos alineamos con la perspectiva de un conjunto importante de directivos y educadores de las escuelas comunitarias, pero también con la perspectiva de varios líderes indígenas, como es el caso de Julio César Pilalumbo, expresidente del Movimiento Indígena de Cotopaxi. Para este último, en particular, el requisito indispensable para que la educación contribuya a propiciar el desarrollo y fortalecimiento de las comunidades indígenas de la zona constituye, sin lugar a dudas, el control directo del proceso educativo por parte de aquellas. ${ }^{6}$

Nos alineamos, también, con aquellos pronunciamientos y textos de la normativa internacional de uso corriente por parte de las organizaciones indígenas, como el Convenio 169 de la Organización Internacional del Trabajo (1989) y la Declaración de las Naciones Unidas sobre los derechos de los pueblos indígenas (2008), que abogan y defienden el derecho de los pueblos indígenas para definir, controlar y gestionar su educación, como un medio para asegurar la pertinencia de aquella.

Tres son los factores que, desde nuestra perspectiva, contribuirían a asegurar un nivel adecuado de control de la educación por parte de las

como la única posibilidad de mantener un proyecto educativo coherente con las necesidades de las comunidades y sensibles con su realidad lingüística y cultural.

6 Julio César Pilalumbo, entrevista personal, Latacunga, 21 de mayo de 2015, Latacunga. 
comunidades: el primer factor tiene que ver con el formato institucional del Estado, el segundo hace alusión al modelo de gestión de la educación, y el tercero se refiere a la población beneficiaria y sus niveles de organización e interés por la educación.

En relación al primer punto, se puede señalar que una condición básica para asegurar el control del proceso educativo por parte de la población indígena, es contar con una instancia específica al interior del Estado para hacerse cargo de ella; una instancia controlada por las mismas organizaciones indígenas y con los recursos suficientes, pero también con el poder necesario para sostener las propuestas de educación indígena.

Consideramos que el modelo por el que se apostó en el Ecuador durante las décadas los 80 y los 90 en materia de organización institucional fue interesante, pues la creación de una jurisdicción propia y diferenciada para la educación indígena, en manos de las organizaciones indígenas, definitivamente contribuyó a crear un espacio idóneo para su desarrollo y fortalecimiento, más aun, en un momento en que las iniciativas de educación indígena eran poco conocidas y menos aún valoradas por la población no indígena e inclusive por la misma población indígena.

El problema, a nuestro entender, no tuvo que ver tanto con el modelo institucional por el que se optó, sino con el hecho de que aquello se dio en condiciones de total asimetría, lo que terminó provocando que la dirección de educación indígena fuera siempre la instancia con menor apoyo técnico, financiero y político; y dependiente de la buena voluntad de los diferentes ministros de turno, que durante las dos décadas y media de vida de la dirección nunca fueron indígenas y menos aún indígenas ligados a las organizaciones.

Siguiendo la línea de reflexión de De Sousa Santos (2010) y siendo coherentes con las demandas históricas y recientes del movimiento indígena, nos adherimos a la propuesta de una institucionalidad plural y paralela, que contemple instancias de gestión de la educación para los diferentes grupos que coexisten en el país, pero instancias que estén al mismo nivel, cuenten con los mismos recursos, y tengan que coordinar directamente, sin intermediarios, para la definición de aspectos medulares de la educación del país. 
El tema es que, luego de varias décadas de desarrollo de iniciativas de educación indígena en la región, queda claro que es necesario una institucionalidad propia para el desarrollo de la educación indígena, pues la reiterada propuesta de contar con un solo sistema educativo en el que se transversalice el enfoque intercultural, como condición para atender las demandas educativas de la población indígena, no ha surtido efecto en ningún país de la región, incluido el Ecuador en su experiencia de la última década.

El segundo factor tiene que ver con el modelo de gestión de la instancia del Estado encargada de la educación indígena, que es en donde creemos ha radicado otra parte importante del problema en la experiencia ecuatoriana. Desde su nacimiento, la DINEIB y las direcciones provinciales operaron con el modelo de gestión estatal hegemónico de la época, a saber, un modelo de gestión altamente centralizado y homogeneizante que, con el tiempo, les llevó no solo a asumir las responsabilidades y atribuciones educativas que estaban en manos de las comunidades; sino también a estandarizar los procesos sin considerar las particularidades de las experiencias locales y de la población de cada sector, lo cual constituye un fenómeno por demás paradójico, si se considera que la DINEIB fue el resultado de la lucha por el reconocimiento y desarrollo de una educación diferenciada.

La situación anterior plantea la necesidad de pensar en nuevos modelos de organización y gestión de la educación, que creen las condiciones necesarias para que las comunidades y sus organizaciones decidan sobre sus proyectos educativos y generen propuestas idóneas para sus contextos. En esta línea, pensamos que necesariamente se debe apelar a modelos de organización y gestión de carácter descentralizado que se hayan experimentado en el país y en otras latitudes, y que hayan sido exitosos en propiciar la participación de las comunidades y su apropiación de la educación.

Un modelo como el que estamos haciendo referencia tendría que, necesariamente, asentarse en una concepción distinta de la gestión y la participación (De Sousa Santos, 2010), pues ya no se trataría de emitir directrices desde arriba en materia educativa y asegurar y controlar que aquellas se concreten localmente, sino de crear las condiciones necesa- 
rias para que la población defina y actualice sus propuestas educativas y tome a cargo elementos centrales de su gestión.

Dicho modelo de gestión debería, también, estar permeado por una concepción diferente de la diversidad, que no vea como un problema el que las iniciativas educativas de cada localidad sean diferentes a nivel de su propuesta formativa y de gestión, pues como se ha constatado en la historia de las últimas décadas, la propuesta formativa y la forma de organización dependen no solo de las características y dinámicas de vida de la población, sino también de los problemas que enfrentan en el día a día y de los proyectos que estructuran para dignificar su vida.

$\mathrm{Y}$, por último, una tercera condición fundamental para asegurar el control es la organización de la población. La experiencia analizada y también otras experiencias de la región demuestran que no puede haber un proceso de apropiación y control de la educación sin comunidades organizadas, pues el control de aquella demanda de un proceso permanente de seguimiento y retroalimentación por parte de las comunidades, cuando no de intervención directa para tomar los correctivos necesarios.

Cuando hacemos alusión al tema de la organización nos referimos no solo a la organización de cada comunidad por separado, sino también a la organización de las comunidades entre sí que propicie la creación de organizaciones mayores. El rol de estas organizaciones es de fundamental transcendencia para el tema educativo, no tanto por el seguimiento y retroalimentación del proceso educativo cotidiano, que estaría en manos de las organizaciones menores, cuanto para servir de mediadoras con las instancias del Estado encargadas de la educación. El tema central, aquí, es que las organizaciones mayores deben ser fieles a los planteamientos de las comunidades y sus organizaciones, y que no manejen una agenda propia.

Ligado con el punto anterior está el tema del lugar que debe ocupar la educación en el proyecto de las comunidades. La experiencia local y regional muestra, también, que junto con la organización de las comunidades, un aspecto de fundamental importancia es que la educación constituya un elemento central de los proyectos políticos de aquellas tanto en el mediano como en el largo plazo. Con esto, nos referimos al hecho de que la educación pase a ser una demanda central de las colec- 
tividades y que esta sea funcional a las otras dimensiones de su proyecto político. La experiencia del SEIC, en sus primeros momentos, constituye un buen ejemplo de lo anterior, así como también la experiencia de otras iniciativas educativas de la región, como la del Consejo Regional Indígena del Cauca (CRIC, 2004).

Los tres factores arriba analizados plantean pistas para alimentar el debate sobre una problemática que las organizaciones indígenas del país y la región han puesto sobre la mesa de discusión durante las últimas décadas, y a la que la academia ha prestado poca atención: nos referimos a la problemática de la plurinacionalidad y sus implicaciones en la organización y gestión de la educación, dimensión por demás relevante y que abre interrogantes para futuras investigaciones.

\section{Bibliografía}

Assies, W. (1999). Pueblos indígenas y reforma del Estado en América Latina. En W. Assies, V. D. Gemma, \& A. Hoekama, El reto de la diversidad. Pueblos indígenas y reforma del Estado en América Latina (pp. 21-56). México: El Colegio de Michoacán.

Baronet, B. (2012). Autonomía y educación indígena. Las escuelas zapatistas de la Selva Lacandona de Chiapas. Quito: Abya-Yala.

Breton, V., \& Martínez, C. (2015). Políticas de reconocimiento neoliberales y posneoliberales en Ecuador: continuidades y rupturas. Quaderns, 25-49.

CONAIE (2007). Propuesta de la CONAIE frente a la Asamblea Constituyente. Principios y lineamientos para la nueva constitución del Ecuador. Quito: CONAIE.

Conejo, A. (2008). Educación Intercultural Bilingüe en el Ecuador. Alteridad (5), 64-82.

CRIC (2004). ¿Qué pasaría si la escuela...? 30 años de construcción de una educación propia. Bogotá: Fuego Azul.

De Sousa Santos, B. (2010). Refundación del Estado en América Latina. Perspectivas desde una epistemología del Sur. Lima: Instituto Internacional de Derecho y Sociedad y Programa Democracia y Transformación Global.

Díaz-Polanco, H. (1998). Autodeterminación, autonomía y liberalismo. En Autonomías indígenas. Diversidad de culturas, igualdad de derechos (págs. 3-10). Quito: ALAI. 


$\frac{\text { Sebastín Granda Merchán }}{214}$

(2006). Elogio de la diversidad. Globalización, multiculturalismo y etnofagia. México: Siglo XXI.

Dussel, E. (2007). Materiales para una política de la liberación. Madrid: Plaza y Valdés.

González, M. I. (2015). Las escuelas clandestinas en Ecuador. Raíces de la educación indígena intercultural. Revista Colombiana de Educación, (69), 75-95.

Grijalva, A. (2008). El Estado Plurinacional e Intercultural en la Constitución Ecuatoriana del 2008. Ecuador Debate, (75), 49-62.

Guerrero, A. (1994). Comentario al libro de Jorge León: De campesinos a ciudadanos diferentes. El Levantamiento indígena. Quito: CEDIME.

Guerrero, P. (1992). El saber del mundo de los cóndores. Identidad e insurgencia de la cultura andina. Quito: Abya-Yala.

Hale, C. (2007). ¿Puede el multiculturalismo ser una amenaza? En M. Lagos, \& P. Calla, Antropología del Estado. Dominación y prácticas contestarias en América Latina (pp. 285-436). La Paz: INDH y PNUD.

Herrán, J. (2008). Testimonio de las escuelas indígenas del Quilotoa. En MIC, DIPEIB-C, \& IEE, Primera convención internacional de educación intercultural bilingüe de Cotopaxi. Memoria. Quito: Instituto de Estudios Ecuatorianos.

Iñiguez, S., \& Guerrero, G. (1986). Un proceso de educación bilingüe intercultural en las escuelas indígenas del Quilotoa. Tesis para obtener el título de licenciatura en la Universidad Politécnica Salesiana.

Larrea, F. (2010). Estado neoliberal y movimiento indígena: neoindigenismo, biopolítica y representación. Tesis presentada para la obtención del título de maestría en FLACSO Ecuador, Quito.

Lazos, E., \& Lenz, E. (s/a). La educación indígena en el páramo zumbahueño del Ecuador. Demandas, éxitos y fracaso de una realidad. s/l: s/e.

León, J. (2010). Las organizaciones indígenas y el gobierno de Rafael Correa. Iconos, (37), 13-23.

López, L. E. (2019). Interculturalidad y políticas públicas en América Latina. En J. E. González, Multiculturalismo e interculturalidad en las Américas. Canadá, México, Guatemala, Colombia, Bolivia, Brasil, Uruguay (pp. 46-101). Bogotá: Universidad Nacional de Colombia. 
Martínez, C. (2004). Los misioneros salesianos y el movimiento indígena de Cotopaxi, 1970-2004. Ecuador Debate, (63), 235-268. (2007). ¿Es el multiculturalismo estatal un factor de profundización de la democracia en América Latina?: una reflexión desde la etnografía sobre los casos de México y Ecuador. En V. Bretón, F. García, A. Jové, \& M. J. Vilalta, Ciudadanía y exclusión: Ecuador y España frente al espejo (pp. 184-202). Madrid: Catarata.

(2010). The "Citizen's Revolution" and the Indigenous Movement in Ecuador: Re-centering the Ecuadorian State at the expense of social movements. Ponencia presentada en el seminario internacional Estados descentrados: formación y deformación política en los Andes, organizado por FLACSO y Emory University.

Martínez, R., \& Burbano, B. (1994). La educación como identificación cultural y la experiencia de educación indígena en Cotopaxi. Quito: Abya-Yala.

OIT (1989). Convenio 169 sobre pueblos indigenas y tribales en países independientes. Recuperado el 6 de enero de 2015, de Consulta indígena: http://www.consultaindigenamds.gob.cl/qconvenio.html

ONU (2008). Declaración de las Naciones Unidas sobre los derechos de los pueblos indígenas. s/l: Naciones Unidas.

Ortiz, P. (2016). Políticas estatales, territorios y derechos de los pueblos indígenas en Ecuador (1983-2012). En P. Ortiz, I. Narváez, \& V. Bretón, Los desafíos de la plurinacionalidad. Miradas críticas a 25 años del levantamiento indigena de 1990 (pp. 13-84). Quito: Abya-Yala.

Pallares, A. (2007). Contesting membership: citizenship, plurculturalism(s), and the conteporary indígenous movement. En K. Klark, \& B. Marc, Highland indians and the state in modern Ecuador (pp. 139-154). Pittsburgh: University of Pittsburgh Press.

Poaquiza, M. C. (2013). Enfoque pedagógico del Sistema de Escuelas Indígenas de Cotopaxi (SEIC) desde la década de 1970 hasta 1998. Tesis presentada para obtener el título de maestría en la Universidad Andina Simón Bolívar-Ecuador.

Ponce, J. (2010). Políticas educativas y desempeño. Una evaluación de impacto de programas educativos focalizados en Ecuador. Quito: FLACSO.

Rodríguez, M. (2018). Educación intercultural bilingüe, interculturalidad y plurinacionalidad en el Ecuador. Luchas y experiencias del movimiento in- 
dígena: desde Dolores Cacuango hasta la Revolución Ciudadana (Vol. 1). Quito: Abya-Yala y Fundación Pueblo Indio del Ecuador.

Tapia, L. (2007). La igualdad es cogobierno. La Paz: CIDES-UMSA, ASDI-SAREC y Plural Editores.

Torres, V. H. (1992). La escuela india: ¿integración o afirmación étnica? Quito: COMUNIDEC.

Van Cott, D. L. (2000). The friendly liquidation of the past: The politics of diversity in Latin America. Pittsburgh: University of Pittsburgh Press.

Wade, P. (2000). Raza y etnicidad en Latinoamérica. Quito: Abya-Yala.

Walsh, C. (2009). Interculturalidad, Estado, sociedad. Luchas (de)coloniales de nuestra época. Quito: UASB, Abya-Yala. 